\title{
An Enhanced-Received Signal Strength Technique for Estimating Mobile Station Position in Wireless Sensor Networks
}

\author{
Adekunle A. Adeyelu \\ Department of Mathematics \& \\ Computer Science \\ Benue State University, Makurdi
}

\author{
Onaji J. Onah \\ Department of Mathematics \& \\ Computer Science \\ Benue State University, Makurdi
}

\author{
Iwuese J. Orban \\ Department of Mathematics \& \\ Computer Science \\ Benue State University, Makurdi
}

\begin{abstract}
Techniques to track an object from a remote location given certain information have been required for several hundred years. Existing outdoor techniques to locate a Mobile Station (MS) within a cellular network require optimization both in terms of accuracy and latency. In this paper, an enhanced Mobile Station Positioning (MSP) model for Wireless Sensor Networks was developed and its performance was appraised using accuracy and latency metrics in line with Received Signal Strength (RSS) procedure. This model used the strength of the signal received at four Base Stations (BS) positioned within the neighborhood of the MS to locate the MS. The mathematical model was formulated using circles equation and Taylor's series expansion. The estimated position of the MS was calculated using Linear Least Square (LLS) solution iteratively. The result showed that the model located the MS within error distances of $199 \mathrm{~m}$ for $67 \%$ and $339 \mathrm{~m}$ for $95 \%$ of the time it was deployed. This result outperformed the RSS technique using three BS which located the MS within $256 \mathrm{~m}$ at $67 \%$ deployment and $368 \mathrm{~m}$ at $95 \%$ of the time the model was used. This gave approximately $15 \%$ improvement in accuracy. Simulation results also revealed that the latency experienced when the BSs were increased from three to four increased by $13.65 \%$ ( 0.024 seconds). It can be concluded that increasing the number of BSs from three to four gave a significant better accuracy in locating a MS within the BSs.
\end{abstract}

\section{General Terms}

Mobile Station Positioning techniques,

\section{Keywords}

Mobile Station, Base Station, Received Signal Strength.

\section{INTRODUCTION}

In the last few decades, wireless networks have been a key part of digital communication and indeed a revolutionary pattern shift that enabled multimedia communications between persons and devices from any location. Cellular communication systems is a type of mobile wireless communication system accomplished using Global System for Mobile communications (GSM) devices which can either reside in a specific network area (Cell) or roam from one cell to another during communication. One of the major issues confronting GSM today is finding the accurate position of a Mobile Station (MS) or GSM device within a GSM network. Any technique deployed to find the exact or approximate location of an MS within a network is known as Mobile Station Positioning (MSP) technique.
Schemes to locate a remote object with respect to a known location have been needed for several hundred years. According to [1], these methods find useful applications in surveying of roads, ranging targets for arms, and in making maps. In the last couple of years however, the number of MS users have constantly been on a dramatic increase. This has also put the number of applications that require location information in a speedy growth and MSP has become an interestingly popular research area [2].

In the world of mobile computing however, the object to be traced is usually the MS. A key drive for the development of MSP techniques to support Location Based Services (LBS) in cellular networks was the need to trace the source of Emergency calls (E-911) made from MS [3]. Presently this initiative has exceeded E-911 scope and is now being applied in location sensitive billing, marketing, location of nodes in a distributed sensor network and users in a social networking application, intelligent traffic system, weather applications just to mention a few.

There are many existing single techniques that have been proposed to estimate location of MS. Examples include Global Positioning System (GPS), Angle of Arrival (AOA), Received Signal Strength (RSS), Time of Arrival (TOA), Time Difference of Arrival (TDOA), Signal Attenuation Difference of Arrival (SADOA) and Cell Identification methods (Cell-ID). Other hybrid techniques like RSS/TDOA, TOA/AOA, AOA/TDOA, and SADOA/TDOA also exist. They combine their strength to outperform the individual techniques that make up the hybrid. Since this is the case, it is only logical that a hybrid technique would deliver a better result if its constituent technique is enhanced to give a better result. Consequently, the authors are motivated to enhance the RSS in this study.

The Federal Communications Commission (FCC) of the United States of America which is the body that regulates telecom released its accuracy standard in 2001 compelling all MSP service providers to comply with. The FCC accuracy requirement states that $67 \%$ of readings produced by any given MSP technique should be within 100 meters of the MS and $95 \%$ of the readings within 300 meters of the MS [4], [2]. The aim of every MSP technique therefore is to meet the FCC accuracy requirement without necessarily consuming too much infrastructure or network resources in the process. The problem lies in the fact that the accuracy of the existing (Network-based) outdoor positioning methods is insufficient as they have not met the accuracy requirement of the FCC especially under the Non Line of Sight (NLOS) environments. 
The RSS technique has the advantage of simplicity of calculation (in terms of algorithm) and is cheaper to implement (it does not require extra hardware to be installed) but not preferred choice in terms of accuracy due to attenuation suffered by signals as they travel from MS to BS and vice versa. This study proposes an enhanced RSS model to locate a MS within a Wireless Sensor Network by improving on the work done by [2] who used three BS. This research made use of measurements from four BSs to improve accuracy of the previous technique. Each RSS reading produces a circle on which the MS may be found. This increment in BSs would definitely lead to complexity in algorithm and latency issues. This paper further investigated the latency caused by the additional BS to see if the solution is viable. The rest of the paper is as follows. Section 2 discusses some key related works by other authors, in section 3, the RSS mathematical model is derived, the methodology for the model is described in detail, and the Algorithm is shown. In section 4, the accuracy and latency results are presented and discussed. Based on these results, conclusions are made in 5 . The paper closes by suggesting future works in section 6 .

\section{LITERATURE REVIEW}

Tracking the location of objects started with the unification of the GPS technology with mobile internet. This made outdoor positioning realize great success in map navigation, people location, object tracking, etc. However, GPS technology meets its frustration in indoor applications because of the great attenuation of the satellite signal and multipath effect caused by the obstruction from buildings and the complex indoor environment [5].

There are two different approaches in position estimation techniques as reported in [6]: single positioning techniques and hybrid techniques. The single techniques include AOA, TDOA, TOA, and RSS just to mention a few. The hybrid techniques for position estimation includes TOA/RSS, TDOA/RSS, TDOA/AOA, and AOA/TOA among many others. Literatures prove that hybrid techniques tend to outperform single schemes. [7], offered a new hybrid technique to locate the MS in urban area using the received signal strength to estimate the time delay and match it with the measured signal time of arrival. Their hybrid technique increased the accuracy and reduced the multi-path signaling influence which affect both TOA and RSS techniques. A new calculation method containing three distance equations was presented to decide the location of MS based on the BSs coordinates. This distance equation was used in this work (to improve on RSS). [8] Proposed a novel indoor localization scheme based on curve fitting and location search. They got $20 \%$ improvement in accuracy of RSS compared with the classical fingerprinting and lateration-based algorithms. [9], made use of the multi-path signaling effect to improve the accuracy of the distance between the MS and the BS. A new path loss formula for computing the distance between the MS and a BS was also presented.

[2], proposed a RSS/TDOA location method using three BSs. Based on their model a RSS measurement, which is the same as the strength of a received BS signal is used to calculate the distance between the MS and BSs. The RSS technique estimates this distance based on the relationship between the signal level and the travelled distance. RSS technique even though it is easy and low-cost technique to implement, its accuracy is not good enough due to the complex transmission equipment. Hence the need for more efforts to improve on
RSS. According to [2], and [6] increasing the number of BSs would be even more beneficial in terms of accuracy.

\section{RECIEVED SIGNAL STRENGTH}

\subsection{Mathematical Model}

In the nonexistence of measurement error, the RSS or received power at the $\mathrm{BS}_{\mathrm{i}}$, denoted by $P_{i}^{r}$, can be demonstrated as in [10]

$$
P_{i}^{r}=K_{\mathrm{i}} \cdot \frac{P_{i}^{t}}{D_{i}^{a}}, \quad \mathrm{i}=1,2, \ldots, \mathrm{N}
$$

where $P_{i}^{t}$ is the transmitted power, $K_{\mathrm{i}}$ is a constraint that accounts for all factors which affect the received power, including the antenna height and antenna gain, and $\mathbf{a}$ is the propagation constant. In free space; $a=2$, but in some suburban and urban places, a can differ from 3 to 6 , for simulations purpose, take $a=4$.

The model in Figure 1 is deployed and the initial guess $\left(x_{0}, y_{0}\right)$ is taken as the center of the cell (polygon) which hosts the BS nearest to the MS.

In the occurrence of measurement error however, the measurement error term $n_{i}$ is added. Hence, (1) can be modeled as

$$
P_{i}^{r}=K_{\mathrm{i}} \frac{P_{i}^{t}}{D_{i}^{a}}+n_{i}, \quad \mathrm{i}=1,2, \ldots, \mathrm{N}
$$

From (2), the range measurements based on the RSS data with the use of the known $\left\{P_{i}^{t}\right\}$ and $\left\{K_{\mathrm{i}}\right\}$, denoted by $\left\{D_{i}\right\}$, are determined as

$$
\begin{aligned}
& D_{i}^{a}=K_{\mathrm{i}} \frac{P_{i}^{t}}{P_{i}^{r}}+n_{i}, \\
& D_{i}=\left[\left(x-x_{i}\right)^{2}+\left(y-y_{i}\right)^{2}\right]^{a / 2}+n_{i}, \\
& \mathrm{i}=1,2, \ldots, \mathrm{N} \\
& \text { For } a=4, \text { then } \\
& D_{i}=\left[\left(x-x_{i}\right)^{2}+\left(y-y_{i}\right)^{2}\right]^{2}+n_{i}, \\
& \mathrm{i}=1,2, \ldots, \mathrm{N}
\end{aligned}
$$

With measurement errors, the LOP derived from linearizing (4) using Taylor series expansion about the point $\left(x_{0}, y_{0}\right)$ has the form

$$
\begin{aligned}
& D_{i}=\left[\left(x-x_{i}\right)^{2}+\left(y-y_{i}\right)^{2}\right]^{2}+n_{i}, \\
& \mathrm{i}=1,2, \ldots, \mathrm{N} \\
& D_{i}-n_{i}=\left[\left(x-x_{i}\right)^{2}+\left(y-y_{i}\right)^{2}\right]^{2} \\
& \quad \mathrm{i}=1,2, \ldots, \mathrm{N}
\end{aligned}
$$

This yield;

$$
\begin{aligned}
& \left(D_{i}-n_{i}\right)^{1 / 2}=\left(x-x_{i}\right)^{2}+\left(y-y_{i}\right)^{2}, \\
& \mathrm{i}=1,2, \ldots, \mathrm{N}
\end{aligned}
$$

Set

$G(x, y)=\left(x-x_{i}\right)^{2}+\left(y-y_{i}\right)^{2}-\left(D_{i}-n_{i}\right)^{1 / 2}=0$

Taylor series expansion of $G(x, y)$ about the point $\left(x_{0}, y_{0}\right)$ 
is given by

$G(x, y)=M\left(x_{0}, y_{0}\right)+\frac{\partial \mathrm{M}(x, y)}{\partial x} \downarrow_{x=x_{0}}\left(x-x_{0}\right)+$

$\frac{\partial \mathrm{M}(x, y)}{\partial y} \downarrow_{y=y_{0}}\left(y-y_{0}\right)+$ Higher Terms

Hence;

$G\left(x_{0}, y_{0}\right)=\left(x_{0}-x_{i}\right)^{2}+\left(y_{0}-y_{i}\right)^{2}-\left(D_{i}-n_{i}\right)^{1 / 2}$

$\frac{\partial \mathrm{G}(x, y)}{\partial x} \downarrow_{x=x_{0}}\left(x-x_{0}\right)=2\left(x_{0}-x_{i}\right)\left(x-x_{0}\right)$

$\frac{\partial \mathrm{G}(x, y)}{\partial y} \downarrow_{y=y_{0}}\left(y-y_{0}\right)=2\left(y_{0}-y_{i}\right)\left(y-y_{0}\right)$

This yield;

$G(x, y)=\left(x_{0}-x_{i}\right)^{2}+\left(y_{0}-y_{i}\right)^{2}-\left(D_{i}-n_{i}\right)^{1 / 2}$

$+2\left(x_{0}-x_{i}\right)\left(x-x_{0}\right)+2\left(y_{0}-y_{i}\right)\left(y-y_{0}\right)$

$=\left(x_{0}-x_{i}\right)^{2}+\left(y_{0}-y_{i}\right)^{2}-\left(D_{i}-n_{i}\right)^{1 / 2}+$

$2\left(x_{0}-x_{i}\right)(x)-2\left(x_{0}-x_{i}\right)\left(x_{0}\right)+$

$2\left(y_{0}-y_{i}\right)(y)-2\left(y_{0}-y_{i}\right)\left(y_{0}\right)=0$

where:

$n_{x i}=\left(x_{0}-x_{i}\right)$

$n_{y i}=\left(y_{0}-y_{i}\right)$

$g_{i}=\left(x_{0}-x_{i}\right)^{2}+\left(y_{0}-y_{i}\right)^{2}$

Reorganizing (10) produces;

$n_{x i} \mathrm{x}+n_{y i} y=\frac{1}{2}\left[\left(D_{i}-n_{i}\right)^{1 / 2}-g_{i}+2 n_{x i} x_{0}+2 n_{y i} y_{0}\right]$

Express the set of linear equations in matrix form,

$\mathrm{A} \hat{X}_{\mathrm{MS}}=\mathrm{B}$

where

$\begin{aligned} \mathrm{A} & =\left[\begin{array}{ll}n_{x 1} & n_{y 1} \\ n_{x 2} & n_{y 2} \\ n_{x 3} & n_{y 3} \\ n_{x 4} & n_{y 4}\end{array}\right], \quad \hat{X}_{\mathrm{MS}}=\left[\begin{array}{l}x \\ y\end{array}\right], \\ \mathrm{B} & =\left[\begin{array}{l}\frac{1}{2}\left(\left(D_{1}-n_{1}\right)^{1 / 2}-g_{1}+2 n_{x 1} x_{0}+2 n_{y 1} y_{0}\right) \\ \frac{1}{2}\left(\left(D_{2}-n_{2}\right)^{1 / 2}-g_{2}+2 n_{x 2} x_{0}+2 n_{y 2} y_{0}\right) \\ \frac{1}{2}\left(\left(D_{3}-n_{3}\right)^{1 / 2}-g_{3}+2 n_{x 3} x_{0}+2 n_{y 3} y_{0}\right) \\ \frac{1}{2}\left(\left(D_{4}-n_{4}\right)^{1 / 2}-g_{4}+2 n_{x 4} x_{0}+2 n_{y 4} y_{0}\right)\end{array}\right]\end{aligned}$
The LLS result is given by

$\hat{X}_{\mathrm{MS}}=\left(A^{T} A\right)^{-1} A^{T} \mathrm{~B}$

\subsection{Methodology}

Matlab 2015a simulation tool was used for the simulation. Figure 1 shows that areas on the Cartesian plane were divided into hexagonal cells. Four arbitrary points $\left(x_{1}, y_{1}\right),\left(x_{2}, y_{2}\right)$, $\left(x_{3}, y_{3}\right)$ and $\left(x_{4}, y_{4}\right)$ were chosen to represent the various BS coordinates, $B S_{1}, B S_{2}, B S_{3}$ and $B S_{4}$. The MS was placed in a known initial position $\left(x_{0}, y_{0}\right)$ between the 4 BSs. The coordinate where the MS was placed (arbitrarily) is $(8,6)$. The exact distances from the MS position to the various BS was also calculated and are represented as D1, D2, D3 and D4 respectively. A zero mean Gaussian random variable is generated as the noise value (in decibel per meter) and subtracted from the value of the signal strength received at each BS. This noise value further fluctuates the distance calculated between the MS and each BS coordinate. This would yield new distance values D11, D22, D33 and D44. This distance values were then used as radii to produce circles each having its center at the corresponding BS. The point of intersection of these circles $\left(x_{0}, y_{0}\right)$ gives the initial estimate for the MS position. This initial distance estimates were used to form a set linear equations (using the equation of a circle). Taylor's series expansion was applied on these distance equations (this is usually a first-level refinement toward a credible location estimate). The resulting set of linear equations yielded matrices and the LLS solution was used to solve the matrices iteratively to further enhance the solution. The iteration ends when further iteration would yield no significant improvement in accuracy. To evaluate the performance of the proposed technique, as illustrated in Figure 1. The received signal at each BS based on the generated noise was used for the MS location calculation. The location performance was assessed in terms of the value of the distance error (Root Mean Square Error) defined by:

$E_{d}=\sqrt{\left(x_{M S}-\hat{x}_{M S}\right)^{2}+\left(y_{M S}-\hat{y}_{M S}\right)^{2}}$

where $\left(x_{M S}, y_{M S}\right)$ and $\left(\hat{x}_{M S}, \hat{y}_{M S}\right)$ are the actual and estimated MS locations, respectively [2]. After several free runs of RSS location estimation for random noise values between MS and BS, results are presented in section 4 . 


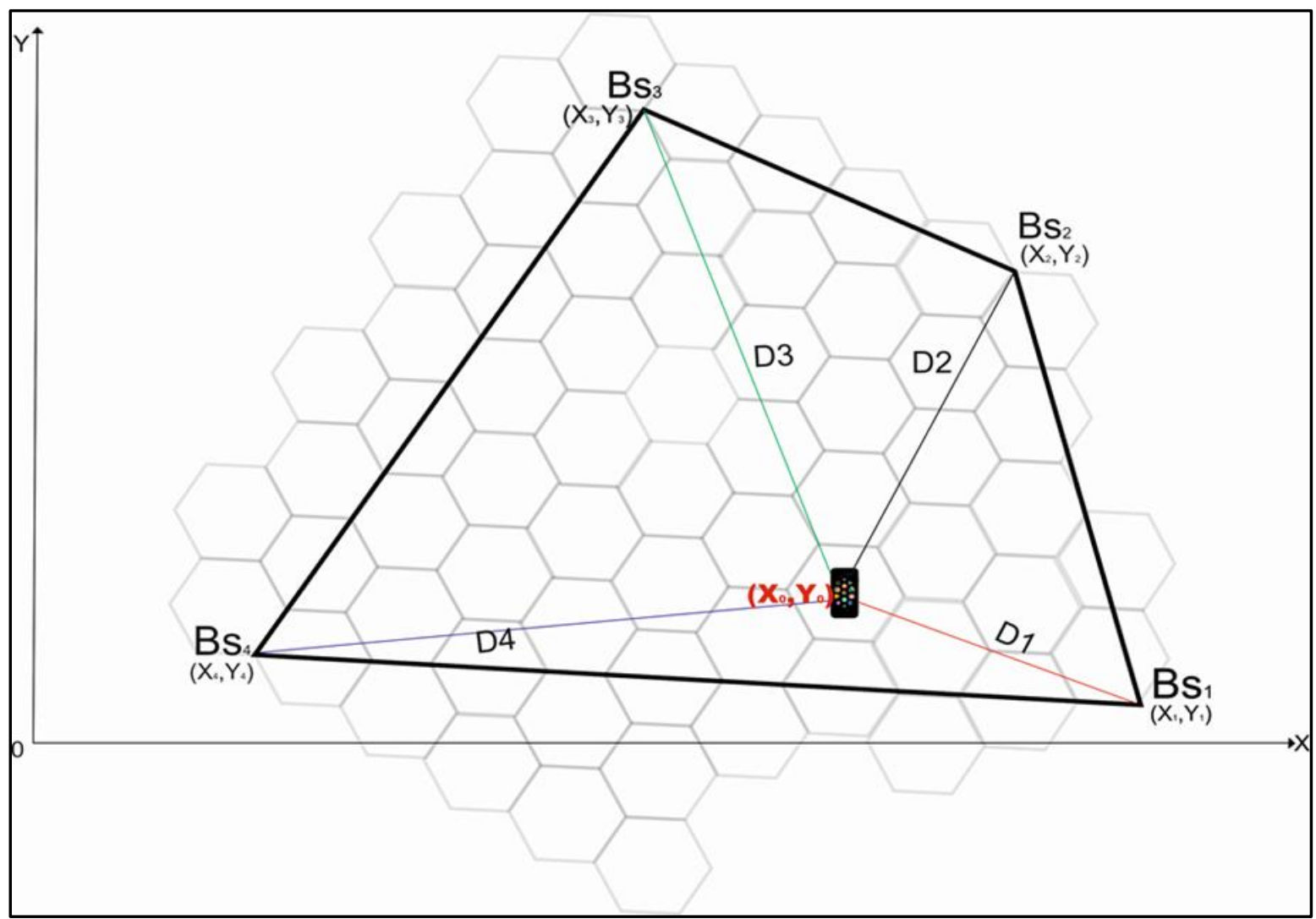

Fig 1: Simulation of the system

\subsection{Algorithm of the System}

The algorithm of the RSS model is given in this section. Some assumptions were made as stated.

\subsubsection{Overall Algorithm of the System}

PRO: xn,yn are the coordinates of the nth BS. The mobile equipment to be located is assumed to be a Mobile Phone object (MS) with the following properties. MS identity (imsi), Serving BS of the MS (sbs), a list of neighboring BSs (nbsi,j) where $1<=\mathrm{I}<=6$ and $1<=\mathrm{j}<=2$, a list of neighboring sorted BSs (nsbsi,j) where $1<=\mathrm{i}<=4$ and $1<=\mathrm{j}<=2$, The BSs have the following nested properties: signal strength, Base Station Code (cid), latitude (x), longitude (y).

POST: This algorithm calculates the location of a Mobile Station Xms using the parameters in PRO and the formula in equation (12).

Step 1: Start

Step 2: Identify the calling Mobile Station Object (MS)

Step 3: Get MS.sbs

Step 4: Set $\mathrm{i}=1$

Step 5:Repeat Step 6 to 8 while $\mathrm{i}<=6$

Step 6: set nbs[i,1] =

getSignalStrengthDBM(MS.getNextnbs());

Step 7: set nbs[i,2] = getCID(MS.getNextnbs ()$)$;

Step 8: set $\mathrm{i}=\mathrm{i}+1$

Step 9: sort nbs with the bubble sort algorithm (descending order) in 3.3.2
Step 10: nsbs[1,1] = getSignalStrengthDBM(MS.sbs);

Step 11: nsbs[1,2] = getCID(MS.sbs);

Step 12: set i to 2

Step 13: Repeat step 14 to 16 while $\mathrm{i}<4$

Step 14: set nsbs $[i, 1]=$ get nbs[i-1,1]

Step 15: set nsbs[i,2] = get nbs[i-1,2]

Step 16: set $\mathrm{i}=\mathrm{i}+1$

Step 17: feed the content of nsbs into equation 12 to produce Xms

Step 18: Exit

\subsubsection{Bubble Sort Algorithm (Descending Order)}

PRO: Assume list is an unsorted array of $n$ elements.

POST: This algorithm returns the sorted version of list in descending order.

Step 1: Start

Step 2: for all elements of list Repeat step 3 to 6

Step 3: if list $[\mathrm{i}]<\operatorname{list}[\mathrm{i}+1]$ Repeat step 4 to 6

Step 4: temp = list [i];

Step 5: list $[\mathrm{i}]=$ list $[\mathrm{i}+1]$;

Step 6: list $[i+1]=$ temp;

Step 7: Exit 


\section{RESULTS AND DISCUSSION}

\subsection{Simulation Data}

The various $E_{d}$ calculated for each iteration was expressed as a percentage of the Cumulative Distribution Function (CDF) and labelled as Location Distance. The result is presented in Table 1 and Table 2 below.

Table 1: RSS 3 BS data set

\begin{tabular}{|c|c|}
\hline \multicolumn{2}{|c|}{ RSS 3BS } \\
\hline Location Distance & CDF (\%) \\
\hline 87.2 & 0.664286 \\
\hline 87.8 & 1.339286 \\
\hline 88.1 & 2.019643 \\
\hline 90.7 & 2.746429 \\
\hline 103.1 & 3.694643 \\
\hline 103.2 & 4.644643 \\
\hline 108.5 & 5.689286 \\
\hline 110.5 & 6.769643 \\
\hline 114.1 & 7.914286 \\
\hline 118.1 & 9.130357 \\
\hline 119.3 & 10.36786 \\
\hline 121.5 & 11.644643 \\
\hline 125.4 & 12.99107 \\
\hline 148.0 & 14.74107 \\
\hline 148.2 & 16.494642 \\
\hline 148.7 & 18.257143 \\
\hline 158.6 & 20.196429 \\
\hline 175.5 & 22.437500 \\
\hline 178.3 & 24.728571 \\
\hline 186.1 & 27.158929 \\
\hline 190.7 & 29.671429 \\
\hline 192.5 & 32.216071 \\
\hline 203.2 & 34.951786 \\
\hline 204.4 & 37.708929 \\
\hline 206.7 & 40.507143 \\
\hline 220.0 & 43.542857 \\
\hline 243.8 & 47.003571 \\
\hline 247.7 & 50.533929 \\
\hline 250.2 & 54.108929 \\
\hline 250.3 & 57.685714 \\
\hline 250.4 & 61.264286 \\
\hline 256.9 & 64.958929 \\
\hline 257.2 & 68.658929 \\
\hline
\end{tabular}

\begin{tabular}{|l|l|}
\hline 261.5 & 72.435714 \\
\hline 264.8 & 76.271429 \\
\hline 306.6 & 80.123214 \\
\hline 322.0 & 84.150000 \\
\hline 337.4 & 88.235714 \\
\hline 352.8 & 90.337500 \\
\hline 368.2 & 96.480357 \\
\hline 370.0 & 100.762500 \\
\hline
\end{tabular}

Table 2: RSS 4 BS data set

\begin{tabular}{|c|c|}
\hline \multicolumn{2}{|c|}{ RSS 4 BS } \\
\hline Location Distance & $\operatorname{CDF}(\%)$ \\
\hline 37.5 & 0.6465517 \\
\hline 39.2 & 1.3224138 \\
\hline 49.7 & 2.1793103 \\
\hline 55.1 & 3.1293103 \\
\hline 55.7 & 4.0896552 \\
\hline 57.1 & 5.0741379 \\
\hline 57.3 & 6.0620698 \\
\hline 62.9 & 7.1465517 \\
\hline 68.8 & 8.3327586 \\
\hline 74.5 & 9.6172414 \\
\hline 77.0 & 10.944828 \\
\hline 77.9 & 12.287931 \\
\hline 85.8 & 13.767241 \\
\hline 94.2 & 15.391379 \\
\hline 98.0 & 17.081034 \\
\hline 100.0 & 18.805172 \\
\hline 102.3 & 20.568966 \\
\hline 103.1 & 22.346552 \\
\hline 109.1 & 24.227586 \\
\hline 115.8 & 26.224138 \\
\hline 122.7 & 28.339655 \\
\hline 126.1 & 30.513793 \\
\hline 126.6 & 32.696552 \\
\hline 134.3 & 35.012069 \\
\hline 139.5 & 37.417241 \\
\hline 148.1 & 39.970690 \\
\hline 151.2 & 42.577586 \\
\hline 153.4 & 45.222414 \\
\hline
\end{tabular}




\begin{tabular}{|l|l|}
\hline 158.6 & 47.956897 \\
\hline 159.6 & 50.708621 \\
\hline 175.0 & 53.725862 \\
\hline 175.6 & 56.753448 \\
\hline 177.8 & 59.818966 \\
\hline 195.0 & 62.939655 \\
\hline 199.1 & 67.200000 \\
\hline 209.3 & 69.481034 \\
\hline 212.7 & 72.774138 \\
\hline 224.0 & 74.150000 \\
\hline 225.8 & 77.594828 \\
\hline 235.7 & 83.227586 \\
\hline
\end{tabular}

\begin{tabular}{|l|l|}
\hline 250.9 & 86.036207 \\
\hline 278.2 & 88.143103 \\
\hline 292.9 & 93.331034 \\
\hline 339.2 & 95.967241 \\
\hline 340.5 & 97.212069 \\
\hline 346.8 & 98.479310 \\
\hline 349.0 & 100.824140 \\
\hline
\end{tabular}

\subsubsection{Accuracy Result}

In this section, the dataset in Table 1 and Table 2 were plotted for easy analysis. The performance of each technique is analyzed based on the CDF of distance error (location error) mainly at $67 \%$ and $95 \%$. This result states by how much this solution approached the FCC accuracy requirement.

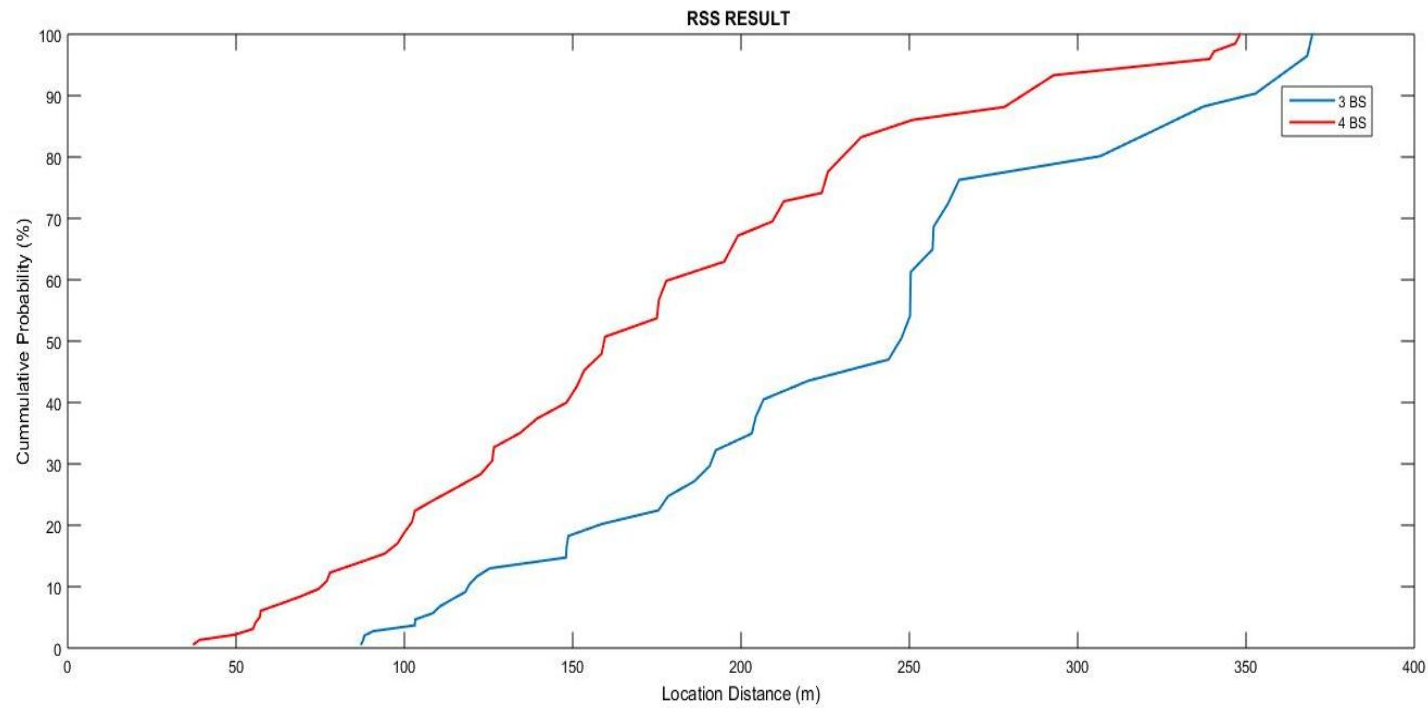

Figure 2: RSS Graph for 3 BS and 4 BS

Figure 2 shows that with measurements from $3 \mathrm{BS}$, at $67 \%$ the MS was located within a distance of approximately $256 \mathrm{~m}$. While at the confidence level of $95 \%$, MS was found within $368 \mathrm{~m}$. In the case of 4 BS, the MS location distance was $199 \mathrm{~m}$ at $67 \%$ deployment, and $339 \mathrm{~m}$ at $95 \%$ of use.

Table 3: Accuracy Result Summary

\begin{tabular}{|c|c|c|c|}
\hline $\begin{array}{c}\text { CDF } \\
(\%)\end{array}$ & $\begin{array}{c}\text { RSS } \\
\text { METHOD }\end{array}$ & $\begin{array}{c}\text { Location } \\
\text { Distance(m) }\end{array}$ & $\begin{array}{c}\text { Enhancement } \\
\text { Level (\%) }\end{array}$ \\
\hline \multirow{2}{*}{67} & $3 \mathrm{BS}$ & 256 & \multirow{2}{*}{22} \\
\cline { 2 - 3 } & $\begin{array}{c}4 \mathrm{BS} \\
\text { (Enhanced) }\end{array}$ & 199 & \multirow{2}{*}{8} \\
\hline \multirow{2}{*}{95} & $3 \mathrm{BS}$ & 368 & \multirow{2}{*}{8} \\
\cline { 2 - 3 } & $\begin{array}{c}4 \mathrm{BS} \\
\text { (Enhanced) }\end{array}$ & 339 \\
\hline
\end{tabular}

As shown in Table 3, the Enhanced technique improved on the 3 BS case by $22 \%$ and $8 \%$ at deployment levels of $67 \%$ and $95 \%$ respectively. This delivered an overall $15 \%$ improvement of the Enhanced RSS over the 3 BS case.

\subsubsection{Latency Result}

In this section, the average time it took to produce the location estimate for each technique is displayed. The result is displayed based on the number of BS used for each calculation. Latency is the delay experienced when producing a location estimate. In this case, the extra time delay experienced when the BS was increased from 3 to 4 gives the latency. A very high latency could make a solution less effective despite a significant increase in accuracy. See Table 5 for comparison.

Table 5: Latency Result

\begin{tabular}{|c|c|c|}
\hline RSS METHOD & 3 BS & 4 BS (Enhanced) \\
\hline $\begin{array}{c}\text { Average Elapsed } \\
\text { Time (Seconds) }\end{array}$ & 0.150224 & 0.173973 \\
\hline Latency Value & \multicolumn{2}{|c|}{0.024} \\
\hline Percentage Latency & \multicolumn{2}{|c|}{$13.65 \%$} \\
\hline
\end{tabular}


As shown in Table 5, the latency value is 0.024 which is a $13.65 \%$ increment in time from 3 to $4 \mathrm{BS}$.

\section{CONCLUSION}

In this study, an enhanced RSS technique was developed to efficiently find the position of a MS in urban and suburban regions. The latency issues that accompanied the increase in number of BS from the conventional 3 to 4 was also investigated. This study showed that the developed technique with 4 BS outperformed the same technique using 3 BS given that no hardware modifications were made to the currently available handsets. Simulation results showed that $67 \%$ of the readings were within 199 meters of the MS and $95 \%$ of the readings were within 339 meters. Hence, there is up to $15 \%$ improvement on the developed technique (using 4 BS) over the same technique using 3 BS. Furthermore, the latency value was 0.024 seconds. Efforts could still be made to reduce or eliminate this latency while sustaining the accuracy. The solution presented in this work is valuable to Communication Engineers, football league industry for goal line technology as well as any distress caller. The extra computation loading at the network end can be calmed by using modern powerful computation machines.

\section{FUTURE WORKS}

The following is recommended for future studies on this work:

i. Efforts should be made to reduce latency without compromising accuracy.

ii. Developed techniques should be stretched to locate more than one MS at a time. This would actually improve latency.

iii. More than two techniques could be merged and their performance evaluated.

iv. Other techniques could be employed to perhaps meet the FCC accuracy requirement.

\section{ACKNOWLEDGMENTS}

Our thanks to the Almighty God who has been by our side all through this research. His grace was sufficient to start and finish this work.

\section{REFERENCES}

[1] T. B. Timothy, H. B. Huub, A. M. Ken and H. P. Wyatt, "A Review of Position Tracking Methods," 1st
International Conference on Sensing Technology, p. 1, 2012.

[2] A.-R. Sharief, K. Yahya and A.-I. Mohammad, "Mobile Station Positioning using Time Difference of Arrival and Received Signal Strength," International Journal of Mobile Communications, October 2012.

[3] R. S. Campos, "Evolution of Positioning Techniques in Cellular Networks, from $2 \mathrm{G}$ to 4G," Wireless Communications and Mobile Computing, p. 1, 12 January 2017.

[4] J. A. d. Peral-Rosado, R. Raulefs, J. A. L'opez-Salcedo and G. Seco-Granados, "Survey of Cellular Mobile Radio Localization Methods: from 1G to 5G," IEEE Communications Surveys and Tutorials, pp. 9-11, 2017.

[5] C. Li, L. Mo and D. Zhang, "Review on UHF RFID Localization methods," JRFID.2019.2924346, IEEE Journal, 2019.

[6] A. D. Gante and M. Siller, "A Survey of Hybrid Schemes for Location Estimation in Wireless Sensor Networks," The 2013 Iberoamerican Conference on Electronics Engineering and Computer Science, 2013.

[7] G. Kbar and W. Mansoor, "Mobile Station Location based on Hybrid of Signal Strength and Time of Arrival," Proceedings of the International Conference on Mobile Business, 2005.

[8] B. Wang, S. Zhou, W. Liu and Y. Mo, "Indoor Localization Based on Curve Fitting and Location Search Using Received Signal Strength," IEEE TRANSACTIONS ON INDUSTRIAL ELECTRONICS, vol. 82, 1 January 2015.

[9] J. Stefanski, "Accuracy Analysis of Mobile Station Location in Cellular Newtorks," Proceedings of the 2nd International Conference on Information Technology, 28 -30 June 2010.

[10] H.-L. Song, "Automatic Vehicle Location in Cellular Communications Systems," IEEE TRANSACTIONS ON VEHICULAR TECHNOLOGY, vol. 43, no. 4, November 1994.

[11] L. Wu, C.-H. Chen and Q. Zhang, "A Mobile Positioning Method Based on Deep Learning Techniques," Electronics ,, vol. 8, no. 59, 2019. 